\title{
Cautious first guidance for prescribing pot
}

$\mathrm{S}$ tart low, go slow and be prepared to say, "No." That's the crux of new College of Family Physicians of Canada guideline for prescribing medical marijuana.

Doctors should only consider the unregulated drug as a last resort treatment for nerve-damage pain caused by conditions such as multiple sclerosis, metastatic cancer, shingles and severe injury, according to the document Authorizing Dried Cannabis for Chronic Pain or Anxiety: Preliminary Guidance.

The college says there is no evidence to support the use of dried cannabis to treat other pain condition, such as fibromyalgia or back pain. Nor is the drug an appropriate therapy for anxiety or insomnia. And doctors should not prescribe it to patients under age 25 , those who are pregnant or breastfeeding, or people with heart disease, lung disease or a history of psychosis or substance abuse.

When it comes to dosage, less is best. Although Health Canada allows physicians to prescribe as much as $5.0 \mathrm{~g}$ of dried cannabis per patient per day, the college advises that "benefit will occur for most patients at considerably lower doses." The guidelines recommend a daily dose of 100-700 mg of cannabis containing less than $9 \%$ delta-9-tetrahydrocannabinol (THC), the principal psychoactive ingredient in marijuana. "We expect that the upper level ... will be on the order of $3.0 \mathrm{~g}$ per day, and that even this level should be considered only in very circumscribed conditions," requiring discussion with colleagues and documentation to support the decision.

The guideline also sets best practices for assessment, monitoring and discontinuing treatment, and include tools for navigating difficult conversations with patients and screening for misuse.

It's a first roadmap to Canada's new and developing medical marijuana landscape but should not be interpreted as an endorsement of the use of the product, says the college. "This situation puts

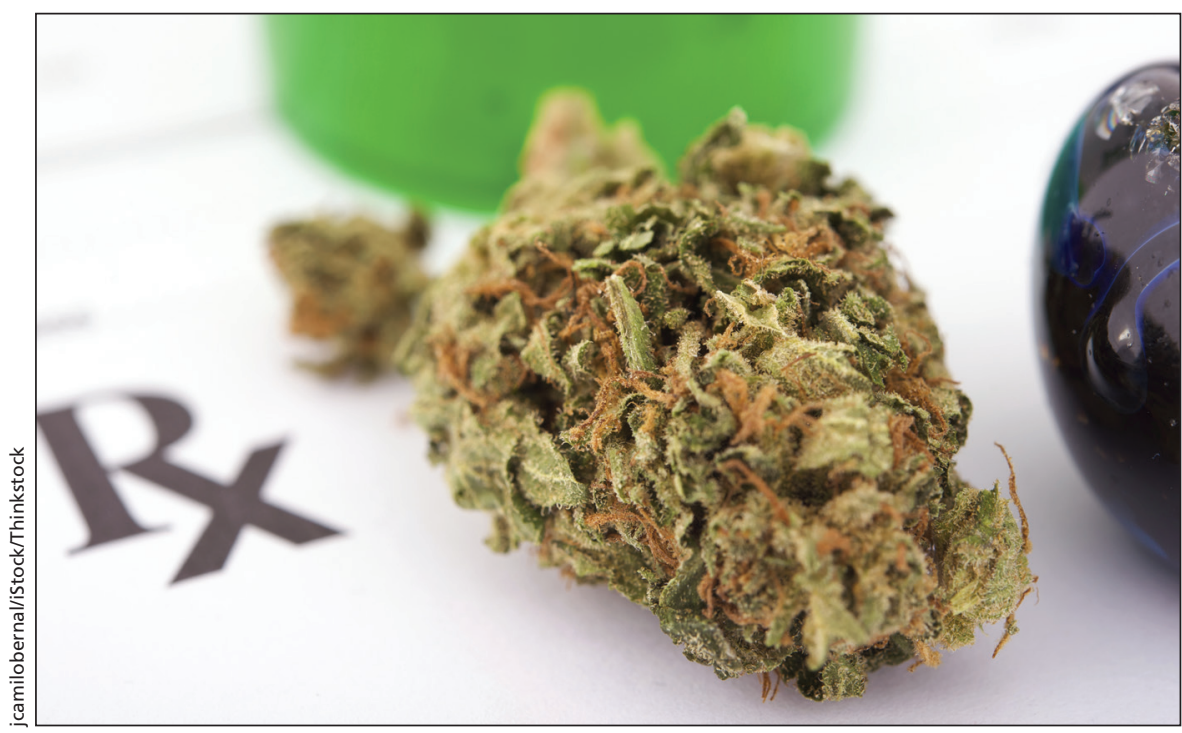

The College of Family Physicians of Canada has created a guideline to help doctors decide when and how to prescribe dried cannabis.

family physicians in a difficult position: we are asked to authorize our patients' access to a product with little evidence to support its use and in the absence of regulatory oversight or approval."

Dr. Chris Simpson, president of the Canadian Medical Association, says the guideline is "appropriately cautious."

"The need for rigorous research still remains," he told CMAJ. "This means adequately powered, well-designed, randomized controlled trials."

But others say the college understates the benefits of marijuana as reported by patients, for many of whom the drug is a last resort after other prescription drugs and therapies prove unsuccessful.

"This overly cautious, paternalistic approach to medical marijuana is physicians trying to protect themselves without really trying to offer an alternative to patients," says Joan Bottorff, director of the Institute for Healthy Living and Chronic Disease Prevention at the University of British Columbia, Okanagan.

As part of the university's Health Effects of Medical Marijuana Project, Bottorff interviewed people who use the drug to cope with a wide range of debilitating chronic illnesses. Participants were clear that the benefits of using cannabis far outweighed the risks or their own concerns; they described it as a "lifeline."

Many of these individuals would not qualify for treatment under the new guideline, says Bottorff. Already, "physicians have made it extremely difficult for these patients," and some participants relied on illegal "compassion clubs" for access to cannabis.

"These were not recreational users in any sense of the word," says Bottorff. "But they couldn't afford to lose their doctors."

Arthritis Society President and CEO Janet Yale says that the paucity of research doesn't reflect a paucity of experience or benefit. "Up to two-thirds of Canadians authorized to use medical cannabis are doing so to manage arthritis pain, and yet there has never been a published study in Canada on medical cannabis and arthritis."

Such anecdotal evidence should spur "all stakeholders in the chronic pain field to fund studies that equip physicians and patients with the knowledge they need to make informed treatment decisions." - Lauren Vogel, CMAJ

CMAJ 2014. DOI:10.1503/cmaj.109-4916 